\title{
Protozoal Infection
}

National Cancer Institute

\section{Source}

National Cancer Institute. Protozoal Infection. NCI Thesaurus. Code C34953.

An infection that is caused by protozoans. 